\title{
Identification of large turbulent structures from simultaneous field and satellite measurements
}

\author{
A. M. Chukharev ${ }^{1,2}$, O. O. Pavlenko ${ }^{1}$ \\ ${ }^{1}$ Marine Hydrophysical Institute RAS, Sevastopol, 299011, Russia \\ ${ }^{2}$ Sevastopol State University, Sevastopol, 299011, Russia \\ E-mail: alexchukh@mail.ru
}

\begin{abstract}
The existence of relatively large formations in developed turbulent flows is an important distinguishing feature and it requires a special approach to modeling. A significant part of the energy carried by large structures significantly affects the processes of interaction between the atmosphere and the ocean, modulating the intensity of heat and momentum flows. Experimental data obtained by contact method at the sub-satellite polygon demonstrate the presence of submesoscale structures in the marine boundary layer, identified by various methods. Wavelet analysis is the most informative method available for analysing in-situ data. The instrument ensemble used on the oceanographic platform, consisting of Sigma, ADCP and Vostok-M instruments, allows us to study the effects associated with both the vorticity of the wind wave field and with larger-scale formations with multi-day periods. A wide set of measured hydrometeorological parameters makes it possible to evaluate the coherence functions between various physical characteristics, including the intensity of turbulent flows. A direct comparison of the results obtained by various methods unfortunately does not give unambiguous conclusions. Both good quantitative and qualitative agreement and differences between the results are observed. The scale of the structures identified by the intensity of turbulence ranges is from 2 to $42-44$ hours (linear scale is $0.3-15 \mathrm{~km}$ ), and scales determined by wind speed and current velocity are over 70 hours. In most cases a high correlation of the detected structures in the marine environment with atmospheric processes.
\end{abstract}

Accepted: 15.09 .2020

DOI: 10.21046/2070-7401-2020-17-6-70-75

\section{Unsteadiness of hydrophysical fields and coherent structures}

Most non-stationary models describing the interaction of the atmosphere and the ocean, except for the seasonal one, usually take into account only the synoptic and (less often) daily variability of the studied characteristics. Their fluctuations on smaller scales, as a rule, are not included to the models. For example, in $[1,2]$ and in a number of others the different models and the different time scales were considered to describe the upper mixed layer of the ocean, but variations in dynamic effects of the order of the inertial period and more were taken into account. Nevertheless there is quite a lot of evidence for the existence of mesoscale and submesoscale spatial structures, which can significantly affect the intensity of turbulent exchange processes both in the boundary layers of the sea and the atmosphere and also its influence on the interaction between two media.

Such relatively large-scale formations in a turbulent flow are quite common and have been described by Townsend [3]. In modern literature, in accordance with experimental results, a coherent structure is defined as "a connected large-scale turbulent liquid mass with vorticity correlated in phase in the entire area of the space occupied by the structure" [4]. The discovery of quasi deterministic 
(coherent) structures, as a widespread phenomenon in many types of flows, has led to a revision of ideas about turbulent motion [5]. Such deterministic structures can carry up to $80 \%$ of the total energy of the turbulent flow. In modern studies, this phenomenon is paid increased attention. Both numerical experiments (LES modelling) and direct measurements usually supported by visualization of flows are carried out. A number of generalizing materials are presented, for example, in reviews [6, 4].

In the last two decades of turbulence research, there has been confidence that the transfer characteristics of momentum, energy, and other quantities in turbulent shear flows are determined for the most part by large-scale vortex motion rather than by small-scale chaotic motion. Moreover, the shape, intensity and scale of such organized movements are different in different flows [4].

Forming of distinct coherent structures, such as convection waves and billows in the atmosphere, and fronts and eddies in the ocean, contributes to the intermittency and variability of exchange processes near the surface [7-9].

Large coherent structures in the ocean and their atmospheric response are observed in the tropical Pacific where with weak winds internal waves contribute to the structure of the surface temperature field and consequently to surface heat fluxes. Studies of coastal upwelling phenomena in the California current have made it possible to describe a system of near-surface vortices and high-energy jets embedded in the main southern surface current. The scale of such features which can be interpreted as quasi-deterministic structures is usually less than $100 \mathrm{~km} \mathrm{[9].}$

The existence of submesoscale structures in the conjugate layers of the atmosphere and ocean should appear as quasi-periodic changes in the turbulent characteristics, which of course leads to changes of interaction intensity. Although strictly speaking the presence of such quasi-periodicities is not sufficient confirmation of coherent structures existence, since point measurements even made simultaneously in different regions of the turbulent flow leave the probability for another interpretation. However, the presence of quasi-periodic changes in both turbulence intensity and various hydrophysical values will be a sign of coherent structures existence in the boundary layers of the sea and atmosphere.

Comprehensive detailed measurements of hydrophysical characteristics and thorough analysis are necessary to achieve a better understanding of the problems of momentum transfer, latent and explicit heat and gas fluxes at the air-sea surface. All these fluxes are controlled by turbulence (including coherent structures) in air and in water, which in turn are strongly influenced by the surface wave field [10]. A more detailed study of the turbulent structure near the surface will help to more accurately take into account the transfer of heat, dissolved elements, gases and pollutants at small scales and in determining the effective transfer coefficients for large-scale problems as subgrid values.

Especially relevant research in situ because the results of experiments in laboratories cannot always be successfully applied to practical tasks.

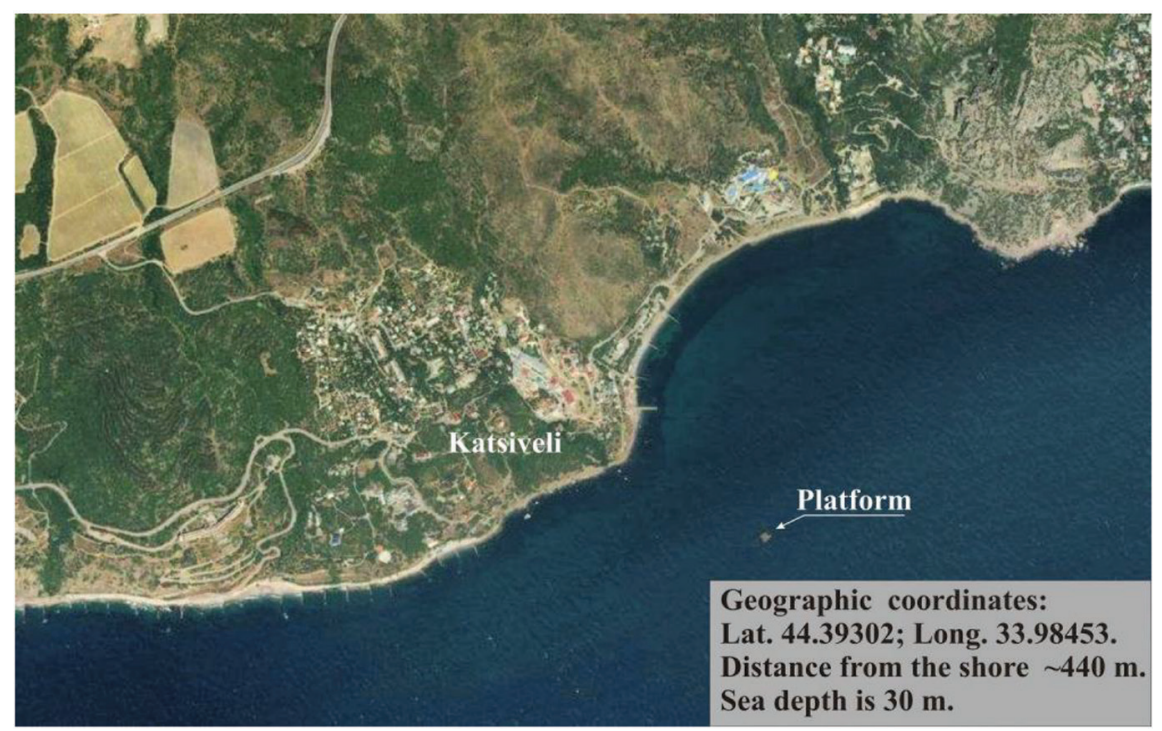

Figure 1. The location of the stationary oceanographic platform of MHI in Katsiveli, Crimea. 


\section{Field measurements of hydrophysical characteristics in the marine boundary layer}

Experimental studies of the turbulent structure in the surface layer were carried out on a stationary oceanographic platform in sub-satellite polygon of Marine Hydrophysical Institute of the Russian Academy of Sciences (MHI) in the Crimea (Figure 1). The set of measuring equipment included a Vostok-M current velocity meter, ADCP, a Sigma-1 turbulimeter, a string wave recorder and a number of others. At the same time, standard weather observations were carried out: wind speed, temperature and humidity and so on. The Sigma-1 measuring complex [11] registers many parameters: $3 \mathrm{D}$ velocity fluctuations: temperature, electrical conductivity, pressure and their pulsation components, that make it possible to estimate the intensity of turbulence. Measurements are taken from the sea side with an appropriate range of flow directions in order to avoid the influence of the platform; the instruments are located at a sufficient distance from the supporting structures.

Thus, the conditions in the measurements area are close to the open sea conditions.

More detailed information about the experiments and methods for estimating the intensity of turbulence is given in [12].

\section{Methods for identifying structures}

Given the importance of relatively large-scale formations in turbulent flows, their detection in the upper sea layer and parameterization are necessary for more adequate description of both the air-sea interaction and substances transport. The following are some methods for identifying structures that have been used with more or less success in practice [13].

(1) Visualization. This method is an essential element of flow analysis. In some cases, it may be the only way to identify structures but in natural conditions it is not always available. A more suitable option may be the PFD (Pseudo Flow Visualization) method. It allows you to simply handle a large amount of data, highlight the large-scale organization of the flow, considering the initial speed data. To obtain information about the flow in space along the axis, the Taylor hypothesis is used.

(2) Study of flow topology. We study the flow field in the vicinity of critical points where the velocity vector $\mathbf{c}=\{u, v, w\}$ disappears. In these singularities, the direction of the flow field is not defined. After linearization of the equation $c=\operatorname{grad}(c)$ using the Taylor series expansion, the eigenvalues are found. The contours separating adjacent singularities (vortices) correspond to the condition that the imaginary part of the root vanishes.

(3) Spectral analysis. This method provides reliable information about the periods of intensity fluctuations in the analysed hydrophysical quantities. It is a well-studied method of analysis and has the widest possible application.

(4) Wavelet analysis. This promising method makes it possible to identify and analyse not only periods of fluctuations in the process intensity, but also its development over time [14]. Guidance on the use of wavelets in geophysical exploration is given in [15]. For our experimental data, this method turned out to be the most informative and convenient. Used wavelet transform

$$
W(a, b)=|a|^{-1 / 2} \int_{-\infty}^{\infty} \xi(t) \varphi^{*}\left(\frac{t-b}{a}\right) \mathrm{d} t,
$$

where $\mathrm{W}(a, b)$ are wavelet coefficients; $a$ is the scale of the wavelet transform; $b$ is the shift along the time axis; $\xi$ is the initial signal; $\varphi$ is the mother wavelet; $t$ is time.

Morlet wavelet was used as the motherboard. In addition to the wavelet coefficients and the global spectrum calculated from them, the autocorrelation function, also calculated from the wavelet coefficients, turned out to be useful [14]:

$$
W c(a, \tau)=\lim _{T \rightarrow \infty} \frac{1}{T} \int_{-T / 2}^{T / 2} W^{*}(a, b) \cdot W(a, b+\tau) \mathrm{d} b,
$$

where $\tau$ is the time delay of the signal. 
In addition to these methods for identifying structures, one can also note empirical orthogonal decomposition, WAG (Window Average Gradient), the method of localization of vorticity peaks, etc. [13].

\section{The results of the analysis and discussion}

The collected arrays of a wide set of hydrophysical values in the marine boundary layer were studied for the existence of large-scale structures with a period of 1 hour or more. The main conclusion is as follows: for all the observed parameters, there are multi-hour periodicities which in our opinion indicate the presence of different-scale structures. Figure 2 shows a histogram of the periods of reliably detected oscillations of the main hydrophysical characteristics during the one operation period (two weeks). Significant periodicities were determined from the global spectrum (calculated using wavelet coefficients) as exceeding the red noise level [15].

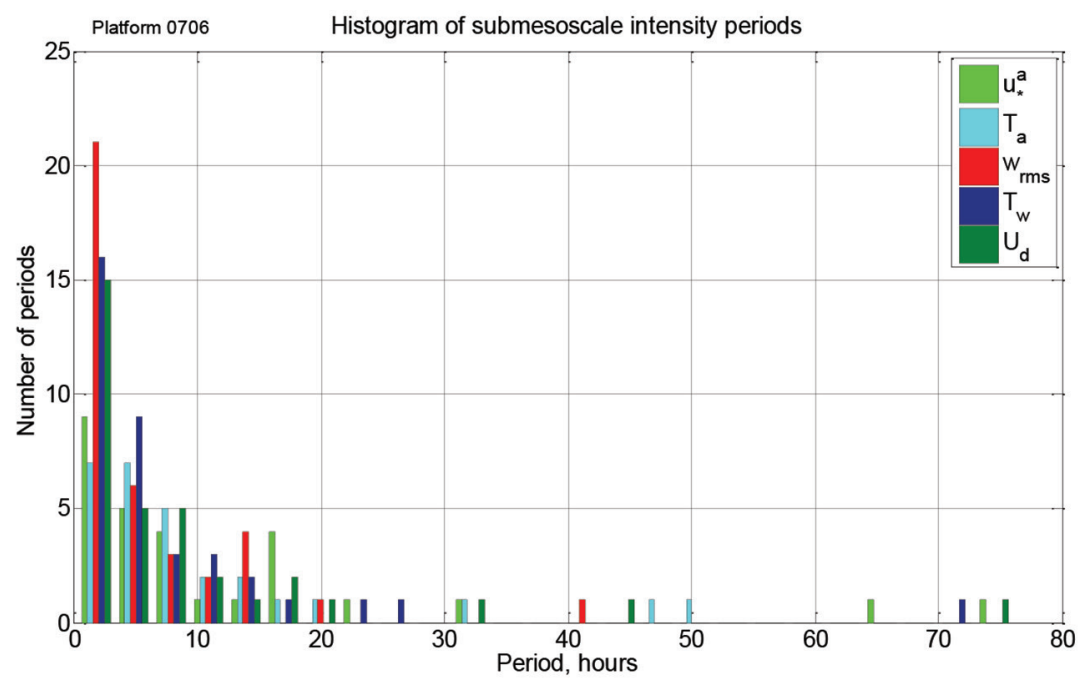

Figure 2. Histogram of detected periodicities distribution in the atmosphere and sea boundary layers in June 2007. Notations: $u_{*}^{a}$ is air fiction velocity; $T_{a}$ is air temperature; $w_{r m s}$ is mean square vertical pulsations of water velocity; $T_{w}$ is water temperature; $U_{d}$ is flow velocity.

The magnitude of the revealed periodicities in all intensity of turbulence, flow velocity, and other hydrophysical quantities was both intraday, lasting from one hour to 18 hours and longer over 70 hours.

Figure 3 shows the global spectrum of vertical velocity pulsations at a depth of $2 \mathrm{~m}$ with intraday periods and the spectrum of red noise. The velocity pulsations were pre-filtered by a high-pass filter with a threshold of $1 \mathrm{~Hz}$ to remove wave components. Here and in the illustrations below, the Morlet wavelet is used as the mother wavelet.
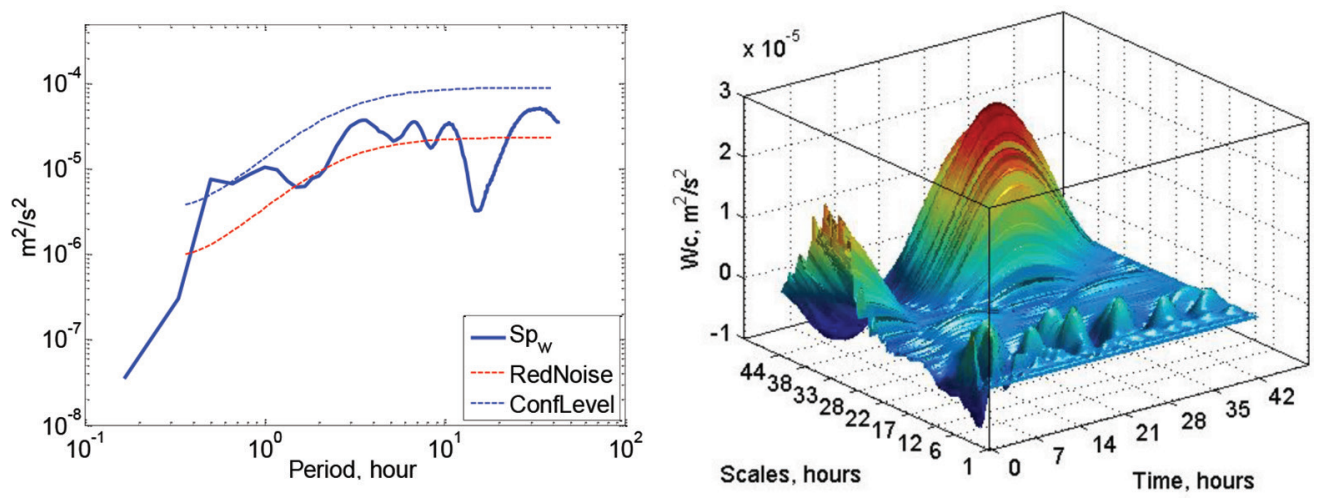

Figure 3. On the left is the global spectrum of rms vertical velocity pulsations at a depth of $2 \mathrm{~m}$, the corresponding spectrum of red noise (lower dashed line) and the $95 \%$ confidence spectrum; on the right is the waveletautocorrelation function of the same series. 
In most cases, the observed structures in the sea subsurface layer and in the atmospheric near-water layer are correlated quite well. However, the coherence level between atmospheric and hydrophysical parameters varied greatly. Structures differing in scale were also observed, which evident to their different origin. Figure 4 shows the autocorrelation functions for the air friction velocity and the root mean square vertical velocity fluctuations in water at a depth of $1 \mathrm{~m}$. It is clearly seen there are both oscillations with the same period ( $\sim 6$ and 12 hours) and differing, almost no diurnal fluctuations in this case in the surface layer although in other observations rather similar patterns have observed.
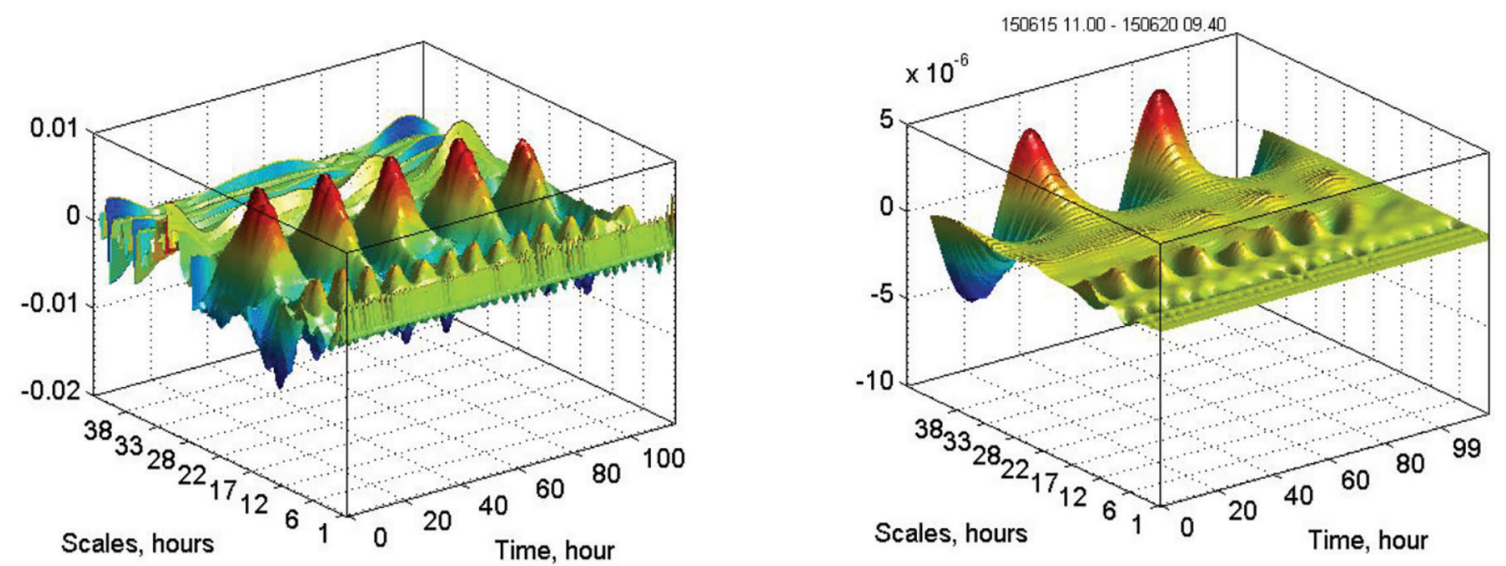

Figure 4. Autocorrelation functions for the friction velocity in air (left) and the rms vertical velocity pulsation in the marine layer at a depth of $1 \mathrm{~m}$. Daily cycling are clearly expressed in the air and are manifested more weekly in water, structures in sea with periods of more than 40 hours are apparently not related to the atmosphere.

Based on the analysis of a large data array from field experiments we can make some generalizations and conclusions.

(1) Known methods for identifying organized structures in turbulent flows can be applied to the analysis of field observations in the marine environment. Unfortunately, a direct comparison of the results obtained by various methods does not give unambiguous conclusions. Both good quantitative and qualitative agreement between the results and differences are observed.

(2) The use of wavelet analysis may be preferable for point measurements and obtaining synchronous information about several hydrophysical parameters.

(3) The hardware set used on the oceanographic platform composed of Sigma, ADCP, and Vostok-M instruments allows one to study effects related to both the vorticity of the wind wave field and larger-scale formations with many-hour and many-day periods.

(4) The scale of the structures identified by the intensity of turbulence is from 2 to $42-44$ hours (roughly from 0.3 to $15 \mathrm{~km}$ ), by wind speed and current velocity scales are reached over 70 hours.

(5) In about $70 \%$ of cases, there is a strong correlation of structures in the marine environment with atmospheric processes, i.e. there is an intense interaction of two environments on large scales.

The presented results will allow us to take into account the presence of submesoscale structures in the developed multiscale models of turbulent exchange and increase their objectivity.

\section{Acknowledgements}

The work was provided as part of state assignment No. 0827-2019-0003.

\section{References}

[1] D’Alessio S.J.D., Abdella K., McFarlane N.A., A new second-order turbulence closure scheme for modeling the oceanic mixed layer, J. Physical Oceanography, 1998, Vol. 24(8), pp. 1624-1641, available at: https://doi.org/10.1175/1520-0485(1998)(028<1624:ANSOTC >2.0.CO;2.

[2] Kundu P.K., A numerical investigation of mixed-layer dynamics, J. Physical Oceanography, 1980, Vol. 10(2), pp. 220-236, available at: https://doi.org/10.1175/1520-0485(1980)010\%3C0220:ANIOML\% 3E2.0.CO;2. 
[3] Townsend A.A., The Structure of Turbulent Shear Flow, Cambridge: Univ. Press, 1956, 315 p., available at: https://doi.org/10.1017/S0022112056210366.

[4] Khlopkov Yu. I., Zharov V.A., Gorelov S. L., Coherent structures in turbulent boundary layer, Moscow: MPhTI, 2002, 268 p.

[5] Fazle Hussain A. K.M., Coherent structures and turbulence, J. Fluid Mechanics, 1986, Vol. 173, pp. 303356, available at: https://doi.org/10.1017/S0022112086001192.

[6] Robinson S. K., Coherent motions in the turbulent boundary layer, Annual Review Fluid Mechanics, 1991, Vol. 23, pp. 601-639, available at: https://doi.org/10.1146/annurev.fl.23.010191.003125.

[7] Seim H., Gregg M., Detailed observations of a naturally occurring shear instability, J. Geophysical Research, 1994, Vol.99(C5), pp. 10049-10073, available at: https://agupubs.onlinelibrary.wiley.com/doi/ abs/10.1029/94JC00168.

[8] Etling D., Brown R.A., Roll vortices in the planetary boundary layer: A review, Boundary-Layer Meteorology, 1993, Vol. 65(3), pp. 215-248.

[9] Flament P., Firing J., Sawyer M., Trefois C., Amplitude and Horizontal Structure of a Large Diurnal Sea Surface Warming Event during the Coastal Ocean Dynamics Experiment, J. Physical Oceanography, 1994, Vol. 24(1), pp. 124-139, available at: https://doi.org/10.1175/1520-0485(1994)024\%3C0124:AAHS OA\%3E2.0.CO;2.

[10] Lumley J.L., Terray E.A., Kinematics of turbulence. Convected by a random wave field, J. Physical Oceanography, 1983, Vol.13(11), 2000-20007, available at: https://doi. org/10.1175/1520-0485(1983)013<2000:KOTCBA>2.0.CO;2.

[11] Samodurov A.S., Dykman V.Z., Barabash V.A., Efremov O. I., Zubov A. G., Pavlenko O. I., Chukharev A.M., "Sigma-1" measuring complex for the investigation of small-scale characteristics of hydrophysical fields in the upper layer of the sea, J. Physical Oceanography, 2005, Vol. 15(5), pp. 311-322.

[12] Chukharev A. M., Multitime scale model of turbulence in the sea surface layer, Izvestiya, Atmospheric and Oceanic Physics, 2013, Vol. 49(4), pp. 439-449, available at: https://doi.org/10.1134/S0001433813040026.

[13] Bonnet J.P., Delville J., Glauser M.N., Antonia R.A., Bisset D. K., Cole D. R., Fiedler H. E., Garem J. H., Hilberg D., Jeong J., Kevlahan N.K.R., Ukeiley L.S., Vincendeau E., Collaborative testing of eddy structure identification methods in free turbulent shear flows, Experiments in Fluids, 1998, Vol. 25, pp. 197-225, available at: ttps://doi.org/10.1007/s003480050224.

[14] Li H., Wavelet auto-correlation analysis applied to eddy structure identification of free turbulent shear flow, JSME Intern. J. Series B, 1997, Vol. 40(4), pp. 567-576.

[15] Torrence C., Compo G. P., A practical guide to wavelet analysis, Bull. American Meteorological Society, 1998, Vol. 79(1), pp. 61-78, available at: https://doi.org/10.1175/1520-0477(1998)079\%3C0061:APGTW A\%3E2.0.CO;2. 